\title{
Unexplained habitual abortion is associated with a reduced endometrial release of soluble intercellular adhesion molecule-1 in the luteal phase of the cycle
}

\author{
Barbara Gaffuri, Lucia Airoldi, Anna Maria Di Blasio, Paola Viganò, Anna Maria Miragoli, Roberta Santorsola \\ and Mario Vignali \\ II Department of Obstetrics and Gynecology, University of Milan and Istituto Auxologico Italiano, Milan, Italy \\ (Correspondence should be addressed to A M Di Blasio, Molecular Biology Laboratory, Istituto Auxologico Italiano, Via Monte Nero 32 , \\ 20135 Milano, Italy)
}

\begin{abstract}
Although the mechanisms causing recurrent spontaneous abortion (RSA) remain frequently speculative, recent evidence indicates that a specific uterine immune-endocrine network plays a pivotal role in the continuation of pregnancy. We have recently demonstrated that an adhesion molecule of the immune system, named intercellular adhesion molecule (ICAM)-1, is markedly expressed at both protein and mRNA levels in endometrial stromal cells and is able to mediate their interaction with lymphoid cells. Moreover, we have shown that the soluble form of ICAM-1 (sICAM-1) can be released by the endometrium in a hormone-dependent manner.

The present study was designed to determine whether surface and/or sICAM-1 expression by cultured endometrial stromal cells could be related to early pregnancy loss in patients with a history of unexplained RSA. Luteal-phase endometrial biopsies were obtained from eight patients who had experienced three or more consecutive unexplained RSAs in the first trimester and 12 control fertile women. Surface ICAM-1 was similarly expressed on luteal-phase endometrial cells obtained from women with and without a history of unexplained RSA. In contrast, the endometrial release of sICAM-1 was significantly lower in abortion-prone patients than in control women.

sICAM-1 is a cytokine-inducible molecule able to interfere with several immunological responses and the reduced levels of the protein shed by the endometrium in patients who have suffered from unexplained RSAs may reflect the presence of an altered immunological environment during the early phases of pregnancy.
\end{abstract}

European Journal of Endocrinology 142 477-480

\section{Introduction}

Recurrent spontaneous abortion (RSA) represents the most common complication of reproductive failure but, despite years of research, information is still lacking on all the etiologies of this event. Chromosomal defects, anatomical anomalies, endocrine disorders and infections are among the causes of RSA. However, in about $60-70 \%$ of cases, abortion remains unexplained and it has long been suspected that immunoregulatory factors may contribute to the pathogenesis of many of the unexplained RSAs (1). An altered local immune regulation during early pregnancy may result in the maladaptation of the fetus and end with RSA. Indeed, the establishment of pregnancy is a complex process composed of a series of developmental phases that, together with steroid hormones, involve local paracrine/autocrine effectors which account for the molecular mechanisms of this enigmatic phenomenon (2).
Human endometrium, in particular, is an active site of cytokine secretion and the blastocyst is able to communicate with the endometrium using the same cytokine receptor language. Cytokines, in turn, can affect the expression of adhesion molecules which may be important for the adhesion phase of trophoblast/endometrium interaction and for leukocyte recruitment, infiltration and function at the implantation site.

Intercellular adhesion molecule (ICAM)-1, a 76$114 \mathrm{kDa}$ surface glycoprotein belonging to the immunoglobulin superfamily, is among the adhesion molecules expressed on human endometrium. Through interactions with $\beta 2$ integrins, ICAM- 1 plays an important role in leukocyte trafficking and cell-cell contact. Expressed basally on many cell lineages of mesenchymal origin, ICAM-1 protein is markedly up-regulated at the transcriptional level by both inflammatory and non-inflammatory cytokines (3). Interestingly, human endometrial stromal cells express surface ICAM-1 throughout the 
entire menstrual cycle and can release its soluble form in a menstrual cycle-dependent manner as levels of the molecule are higher in the proliferative when compared with the secretory phase of the cycle (4). The regulation of synthesis and function of ICAM-1 in endometrial physiology is still to be definitively clarified. However, as the molecule is cytokine inducible and menstrual cycle related, its pattern of expression might mirror the interactions mediated by the cohort of factors acting at the implantation site.

To our knowledge, ICAM-1 expression by endometrial cells has not yet been investigated in the specific assessment of endometrial function and response to cytokine stimulation in patients who have experienced unexplained RSAs. We therefore studied the endometrial levels of surface and soluble ICAM-1 (sICAM-1) in non-conceptional cycles of patients with a history of unexplained RSA and in control women not susceptible to RSA.

\section{Materials and methods}

Eight patients with three to four consecutive miscarriages were studied during one menstrual cycle. Abortion-prone patients had no obvious cause for recurrent miscarriage, including endocrine, genetic, infective, anatomical or other medical complications. None had any autoimmune abnormalities, such as antiphospholipid antibodies, lupus anticoagulant, anticardiolipin and antinuclear antibodies including anti-ribonucleoprotein antigens (Ro/SSA). These patients underwent endometrial biopsy in the luteal phase of the menstrual cycle on postovulatory days $2-13$ as part of their infertility evaluation. Chronological dating was established by evaluation of basal temperature and the onset of the next menstrual period. Patients were included only if chronological dating agreed with histologic dating criteria (within 2 days). The early, mid- and late luteal phases were defined as the periods $2-4,5-9$ and
10-14 days after ovulation respectively. The control group consisted of 12 regularly cycling women undergoing laparoscopy for benign ovarian cysts. All of them had previously delivered at least one child. Histologic examination demonstrated that cysts were dermoid in $60 \%$, serous in $25 \%$ and mucinous in $15 \%$ of the cases. None of these patients had identifiable endometriosis. Endometrial biopsies were performed using an endometrial biopsy curette. The patients were informed that the tissues would be used for research purposes and gave written consent. All the study subjects had regular menstrual cycles and a normal serum level of progesterone $(\geq 10 \mathrm{ng} / \mathrm{ml})$ in the mid-luteal phase; i.e. they did not suffer from any luteal-phase defect. Clinical characteristics of both groups included in this study are presented in Table 1 . The study protocol was approved by the Institutional Review Board of the II Department of Obstetrics and Gynecology, University of Milan, Milan, Italy.

Stromal cell monolayers from endometrial samples were established as previously reported (5-7). With this technique, diffuse and strong cytoplasmatic immunostaining for vimentin is present in nearly all (90\%) cultured stromal cells. For both groups of women, endometrial stromal cultures used for supernatant collection were allowed to proliferate to subconfluence in Ham's F-10 supplemented with $10 \%$ fetal calf serum (FCS; EuroClone, Celbio, Milan, Italy) and antibiotics in a humidified atmosphere of $95 \%$ air and $5 \% \mathrm{CO}_{2}$ at $37^{\circ} \mathrm{C}$. Subconfluence was reached after 8-10 days. To completely eliminate contaminating leukocytes from the cultures, cells were subjected to a single passage that did not affect ICAM-1 expression or shedding as assessed in preliminary experiments. Therefore, cells were harvested by a 10-min incubation in 0.05\% trypsin$0.02 \%$ EDTA solution, counted, resuspended in Ham's F-10 plus FCS and plated at a density of $5 \times 10^{5} / \mathrm{ml}$ in Petri dishes $(1 \mathrm{ml} /$ dish, $35 \mathrm{~mm}$ in diameter) for an additional 48-h period. Finally, medium was collected,

Table 1 Clinical characteristics of patients with unexplained spontaneous abortions and a control group.

\begin{tabular}{|c|c|c|}
\hline & Control group & Patients with RSA \\
\hline Age (mean years \pm S.E.M.) & $31.5 \pm 1.27$ & $32.3 \pm 1.8$ \\
\hline $\begin{array}{l}\text { No. of RSA patients } \\
\text { (mean } \pm \text { S.E.M.) }\end{array}$ & 0 & $3.1 \pm 0.12$ \\
\hline Parous (\%) & 100 & 0 \\
\hline $\begin{array}{l}\text { Progesterone serum level } \\
\text { (mid-luteal phase) }\end{array}$ & $\geq 10 \mathrm{ng} / \mathrm{ml}$ & $\geq 10 \mathrm{ng} / \mathrm{ml}$ \\
\hline \multicolumn{3}{|c|}{ Phase of endometrial biopsies } \\
\hline Early secretory & 4 & 2 \\
\hline Mid-secretory & 6 & 5 \\
\hline Late secretory & 2 & 1 \\
\hline Autoimmune abnormalities & Absent & Absent \\
\hline Karyotypic examination & Normal & Normal \\
\hline Hysterosalpingography & Absence of anomalies & Absence of anomalies \\
\hline Cervical cultures & Negative & Negative \\
\hline
\end{tabular}


centrifuged for $5 \mathrm{~min}$ at $400 \mathrm{~g}$ and stored at $-20{ }^{\circ} \mathrm{C}$ until analysis. Cells were used immediately for flow cytometric analysis.

Immunofluorescent staining was performed using an unconjugated murine anti-ICAM-1 (anti-CD54; Immunotech, Marseille, France) followed by a secondary polyclonal goat anti-mouse IgG-FITC as previously described (8). A matched IgG isotype control obtained from murine myeloma followed by the secondary antibody was used to evaluate background fluorescence. Stained cells were examined with a flow cytometer (FACScan; Becton Dickinson \& Co., Mountain View, CA, USA). The quantitative detection of sICAM-1 in cell-free supernatants was performed using a commercially available enzyme-linked immunosorbent (ELISA) kit provided by Bender MedSystem (Vienna, Austria). The unpaired Student's $t$-test was used to test for statistical significance of differences between groups. A probability $<0.05$ was considered as statistically significant. Values are means \pm s.E.M.

\section{Results}

ICAM-1 surface expression was assayed on endometrial stromal cells obtained from patients with and without RSA using immunofluorescence flow cytometry. In both groups, ICAM-1 fluorescence values (376 \pm 36.9 and $435 \pm 34.0$ respectively) and the percentage of ICAM-1positive cells $(47.5 \pm 9.8$ and $60.5 \pm 9.4$ respectively) were similar.

In contrast, as shown in Fig. 1, sICAM-1 levels measured in 48-h medium conditioned by luteal-phase endometrial stromal cells from patients with a history of RSA were significantly lower $(2.4 \pm 1.1 \mathrm{ng} / \mathrm{ml})$ when

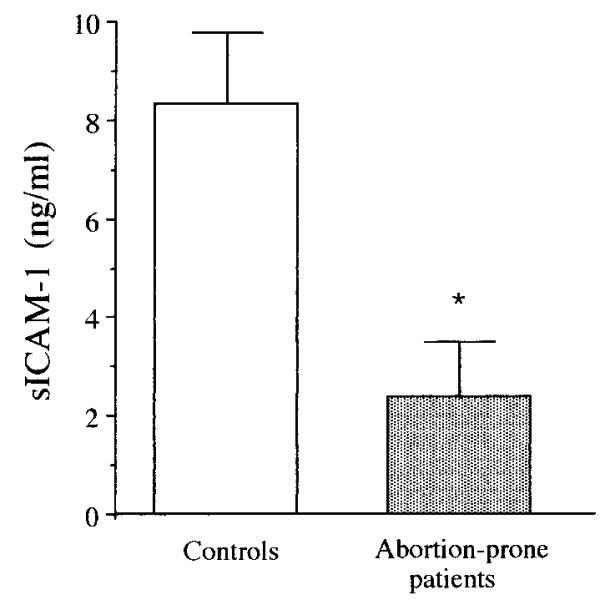

Figure 1 Levels of SICAM-1 released from luteal-phase endometria stromal cells. Endometrial biopsies were obtained from 12 control women and eight patients with a history of unexplained RSA. Forty-eight-hour conditioned media were harvested from cultures containing $5 \times 10^{5}$ cells $/ \mathrm{ml}$ and tested for their content of SICAM-1 by a specific ELISA. Data are expressed as means \pm S.E.M.; ${ }^{*} P<0.01$ vs controls. compared with those obtained in control women $(8.34 \pm 1.4 \mathrm{ng} / \mathrm{ml} ; P<0.01)$. sICAM-1 levels in culture medium alone were $<0.5 \mathrm{ng} / \mathrm{ml}$.

Values of sICAM-1 analyzed as a function of the different periods of the luteal phase in control women were $5.5 \pm 1.4,10.6 \pm 2.4$ and $7.1 \pm 0.1 \mathrm{ng} / \mathrm{ml}$ respectively in early, mid- and late luteal phases. Therefore, as levels of sICAM-1 tended to be higher in the midluteal phase, we also evaluated the potential differences in endometrial sICAM-1 levels considering only midluteal phase endometria. Mid-luteal phase samples from patients with three or more consecutive abortions released significantly lower levels of sICAM-1 when compared with those derived from control women $(3.2 \pm 1.7$ and $10.6 \pm 2.4 \mathrm{ng} / \mathrm{ml}$ respectively; $P<0.05)$.

\section{Discussion}

ICAM-1 is a sialylated glycoprotein comprising five immunoglobulin-like domains, a single transmembrane region and a short cytoplasmic tail. It is a natural ligand for at least two members of the leukocyte integrins, lymphocyte function-associated antigen-1 (LFA-1) and Mac-1, and mediates various cell interactions, including neutrophil extravasion and lymphocyte-mediated cytotoxicity (3). Antibodies to ICAM-1 inhibit leukocyte adhesion to endothelial cells, granulocyte migration through endothelium, antigen-induced lymphocyte proliferation and major histocompatibility complex (MHC) and non-MHC-restricted cytotoxicity (9). A shedding form of this adhesion molecule has lately been identified and has been suggested to act as an antagonistic form of the surface molecule $(9-11)$.

In the present study, sICAM-1 levels produced by luteal-phase endometrial cells derived from normal menstruating women were perfectly in line with our previous reports showing the pattern of the soluble molecule during the menstrual cycle $(4,8)$. Interestingly, the investigation of sICAM-1 concentrations produced by the endometrial cells of non-pregnant patients with a history of unexplained RSA revealed significantly lower values than control women. The reduced release of endometrial sICAM-1 observed in these patients could not be attributed to a significant reduction of the molecule on cell membrane, as evaluation of surface ICAM-1 expression showed a similar profile in both groups of women.

At present, little is known about the biological function of sICAM-1 in endometrial physiology and early pregnancy. The release of this soluble molecule may represent a mechanism for breaking adhesive contacts between cells in order to control cellular adhesivity and rearrangement. Otherwise, the molecule may be effective in controlling lymphocyte-mediated events and in preventing immune recognition in the uterine cavity. In this context, it should be noted that, given the ability of sICAM-1 to interfere with specific immunological responses (9-11), its role may be similar to that of other 
immunoregulatory molecules which are secreted at endometrial and decidual level such as placental protein 14 , progesterone-induced blocking factor and transforming growth factor $\beta 2(1,12)$. It has been suggested that these factors are involved in mediating the inhibition of the maternal immune system during pregnancy and, more interestingly, some of them have been reported to be decreased in women with $\operatorname{RSA}(1,12)$. Whether sICAM-1 is actually associated with these immunomodulatory molecules involved in the implantation process awaits further investigation. If this is the case, it is conceivable that, in habitual aborters, a deficient release of endometrial sICAM-1 in the midluteal phase, which corresponds to the putative 'implantation window', may reflect the presence of an altered immunologic environment for the maintenance of a normal pregnancy.

Our inability to detect a modification of surface ICAM-1 parallel to the decrease in SICAM-1 in patients with unexplained spontaneous abortions is not completely understood. Soluble adhesion molecules are probably formed by cleavage and release of the extracellular domain of the membrane-bound form (13). However, while the surface form mediates adhesive phenomena with LFA-1-bearing leukocytes, the soluble molecule seems to promote de-adhesion and leukocyte migration (3). If the soluble molecule has any role in the recruitment of specific leukocyte populations into the stroma or in the modulation of maternal local immune response in the uterus in the second half of the secretory phase, it is likely that modifications of its levels may contribute to a pregnancy failure.

Finally, it must be considered that several cytokines are able to modulate this adhesion molecule (3). Indeed, specific cytokines, claimed to be crucial for endometrial physiology and embryo implantation, have been shown to induce or enhance endometrial expression of both membrane and soluble ICAM-1. We have recently demonstrated that interleukin-1 $\beta$, which has been suggested to be an important endometrial component for successful implantation, is a potent inducer of sICAM-1 shedding from endometrial cells (8). The protein expression can also be augmented by tumor necrosis factor- $\alpha$ (3), which is physiologically produced at the endometrial level (14). Therefore, quantitative assessments of this protein may be of great value in understanding the interplay of various cytokines and hormones acting at different phases of endometrial function and, most importantly, in detecting any deleterious perturbation of their secretion.

\section{References}

1 Raghupathy R. Th1-type immunity is incompatible with successful pregnancy. Immunology Today 199718 478-482.

2 Simon C, Gimeno MJ, Mercader A, Francés A, Velasco JC, Remohì $\mathrm{J}$ et al. Cytokines-adhesion molecules-invasive proteinases. The missing paracrine/autocrine link in embryonic implantation? Molecular Human Reproduction 19962 405-424.

3 Hayflick JS, Kilgannon P \& Gallatin WM. The intercellular adhesion molecule (ICAM) family of proteins. Immunological Research 199817 313-327.

4 Somigliana E, Viganò P, Gaffuri B, Guarneri D, Busacca M \& Vignali M. Endometrial stromal cells as a source of intercellular adhesion molecule (ICAM)-1 molecules. Human Reproduction 199611 1190-1194.

5 Viganò P, Di Blasio AM, Dell'Antonio G \& Vignali M. Culture of endometrial cells: a new simple technique to completely separate epithelial glands. Acta Obstetrica et Gynecologica Scandinavica 1993 $7287-92$.

6 Di Blasio AM, Pecori-Giraldi F, Viganò P, Petraglia F, Vignali M \& Cavagnini F. Expression of corticotropin-releasing hormone and its R1 receptor in human endometrial stromal cells. Journal of Clinical Endocrinology and Metabolism 199782 1594-1597.

7 Viganò P, Gaffuri B, Somigliana E, Busacca M, Di Blasio AM \& Vignali M. Expression of intercellular adhesion molecule (ICAM)1 mRNA and protein is enhanced in endometriosis versus endometrial stromal cells in culture. Molecular Human Reproduction 19984 1150-1156.

8 Viganò P, Gaffuri B, Ragni G, Di Blasio AM \& Vignali M. Intercellular adhesion molecule (ICAM)-1 is expressed on human granulosa cells and mediates their interaction with lymphoid cells. Journal of Clinical Endocrinology and Metabolism 199782 101-105.

9 Becker JC, Termeer C, Schmidt RE \& Brocker EB. Soluble intercellular adhesion molecule-1 inhibits MHC-restricted specific T cell/tumor interaction. Journal of Immunology $19931517224-$ 7232.

10 Becker JC, Dummer R, Hartmann AA, Burg G \& Schmidt RE. Shedding of ICAM-1 from human melanoma cell lines induced by INF- $\gamma$ and tumor necrosis factor- $\alpha$. Functional consequence of cell-mediated cytotoxicity. Journal of Immunology 1991147 4398-4401.

11 Meyer DM, Dustin ML \& Carron CP. Characterization of intercellular adhesion molecule-1 ectodomain (SICAM-1) as an inhibitor of lymphocyte function-associated molecule-1 interaction with ICAM-1. Journal of Immunology 1995155 3578-3584.

12 Dalton CF, Laird SM, Estdale SE, Saravelos HG \& Li TC. Endometrial protein PP14 and CA-125 in recurrent miscarriage patients; correlation with pregnancy outcome. Human Reproduction 199813 3197-3202.

13 Nash MC, Wade M, Shah V \& Dillon MJ. Normal levels of soluble Eselectin, soluble intercellular adhesion molecule-1 (sICAM-1), and soluble vascular cell adhesion molecule-1 (sVCAM-1) decrease with age. Clinical and Experimental Immunology 1996103 167-170.

14 Laird SM, Tuckerman EM, Saravelos H \& Li TC. The production of tumor necrosis factor $\alpha$ (TNF- $\alpha$ ) by human endometrial cells in culture. Human Reproduction 199611 1318-1323.

Received 15 September 1999

Accepted 10 January 2000 